\title{
Mobile Customer Relationship Management: An Explorative Investigation of the Italian Consumer Market
}

\author{
Giovanni Camponovo *, Yves Pigneur *, Andrea Rangone **, Filippo Renga ** \\ * HEC School of Business, University of Lausanne \\ ** Department of Management, Economics and Production, Politecnico of Milan \\ Giovanni.Camponovo@unil.ch,Yves.Pigneur@unil.ch,Andrea.Rangone@polimi.it,Filippo.Renga@polimi.it
}

\begin{abstract}
Mobile Customer Relationship Management (CRM) services seem to have all the characteristics commonly associated to successful mobile services and have accordingly been predicted among the most promising ones. Yet, the real development of this sector has not been well explored so far, especially in relation to the actual supply of mobile CRM services to the public. The purpose of this paper is to reduce this gap by giving a first snapshot of the current development of the supply of mobile CRM services to consumers taken in the context of the Italian market. In order to do so, it firstly proposes a conceptual framework indicating the relevant aspects to investigate for assessing this kind of environments: the market, value propositions, actors and issues. Then it applies this framework to get an overview of the supply of mobile CRM services in Italy and provides some empirical insight about its current development obtained through an exhaustive survey of the current supply of 750 services from 353 firms.
\end{abstract}

\section{Introduction}

The mobile business industry is rapidly emerging thanks to a set of technologies which potentially enable a large variety of innovative services. In effect, the mobile and personal nature of wireless devices combined with the voice and data transmission capabilities of wireless networks provides a set of unique features such as ubiquity, 24h reachability, localization, personalization dissemination and convenience which are commonly considered the most valuable distinctive advantages upon which mobile services can build their value proposition [11,21, 27].

Among the variety of mobile services, considerable attention has been devoted to mobile marketing and in particular to mobile customer relationship management services. The reason is that the previously mentioned characteristics of the mobile medium does confer it highly valuable and unique peculiarities which can be exploited as a complement to other channels to create and manage personalized customer relationships [15]. In particular, mobile phones have shown to be very personal devices which may provide firms with unrivalled possibilities to build and maintain one-toone relationships with their customers, combined with a large reach, low costs, rapid feedback, constant reachability and localization possibilities.

This paper specifically focuses on mobile Customer Relation Management services (thereafter referred to as mobile CRM services), which deal with managing the customer relationship throughout the entire customer life cycle, hence supporting marketing, sales and after sale service activities. The reason of this choice is twofold.

On the one hand, the mobile medium is well suited to enhance traditional CRM because its very personal character, large reach and interactivity may allow the firm to develop intimate relationships with customers. CRM is indeed among the enterprise applications that generate the most attention with respect to mobility [4].

On the other hand, despite mobile CRM services are becoming a substantial phenomenon, they have insofar not been well explored. In effect, despite there is some empirical research about traditional CRM solutions [9] and mobile marketing services [10] in the Italian market, there is no research which focuses on the offering of mobile CRM services to consumers on the Italian market and their value to the offering firms.

The primary purpose of this paper is therefore to fill this gap by assessing the actual development of the mobile CRM services to consumers landscape with an exploratory analysis. The Italian context is chosen as field of research because it is an advanced market with one of the highest rates of mobile phone ownership in the world, ranking second in Europe in terms of mobile telephony penetration, fifth worldwide in total number of mobile subscribers and having one of the highest per 
capita SMS usage rates in the world [14]. Moreover, the market of content-based mobile services in Italy is very important: for instance the market of music-based services such as ringtones is about 140 million $€$ worth, more than one half that of musical CDs and DVDs [5].

In order to achieve this objective, the paper firstly defines in the next section what we intend with mobile CRM. Then section 3 shows a conceptual framework with the relevant aspects to consider in the analysis of this kind of environments. Section 4 is the central part of the paper: it applies the framework to the mobile CRM landscape and gives the results of our empirical investigation of the actual development of mobile CRM services to consumers in Italy, apprehended through an exhaustive census of the current offer of 750 services proposed by 353 enterprises. Finally, section 5 discusses the major findings of this study and gives ideas about future possible research directions.

\section{A definition of Mobile CRM}

Before undertaking the analysis of the mobile CMR services landscape, it is useful to give a clear definition of what is intended with mobile CRM.

In its traditional form, CRM is a business strategy and systematic approach issued from relationship and one-to-one marketing which is based on the integrated and active management of personalized individualized with customers $[12,16]$.

The principle is to create and maintain personalized relationships with each customer which strengthen the mutual benefit of both parties through individualized, interactive and value-added contacts [26]. The underlying belief is that such personal relationships lead to improved customer acquisition, satisfaction and loyalty, allowing better returns to be achieved. To do so, the firm shall take advantage of every contact with customers to learn more about their needs, preferences and lifetime value, so as to devise suited ways to serve them with possibly tailored solutions and devote them an appropriate amount of attention [23].

It must be emphasized that CRM is not confined to sales management but spans over the entire customer life cycle, covering marketing, sales and after sale service activities $[19,30]$. In fact, the portfolio of CRM services addresses various cross-functional processes such as campaign management, customer contact and lead management, offer creation and delivery, contract management, customer complaint and retention management as well as after sales service [12].

One primordial capacity needed for implementing a CRM strategy is the ability to communicate with customers on an individual basis [23]. For that reason, mobile technologies represent an appealing additional channel which can complement the existing channels. Among the advantages of the mobile channel which are highly relevant to CRM are the personal character of mobile devices which allows an individual customer reach, the interactivity brought by its quick message delivery and response, its reachability and ubiquity. It is the only medium enabling a spontaneous, interactive, direct and targeted interaction with customers, any time, anywhere [20]. This makes it a valuable channel despite the drawbacks of mobile devices (e.g. limited screen, memory, computing power and I/O resources) and networks (e.g. high cost, limited bandwidth and low amount of data conveyed through SMS). For that reason, the future CRM solutions is envisaged to combine traditional, Internet and mobile channels [17].

We thus define mobile CRM services as those that 1) aim at nurturing customer relationships, acquiring or maintaining customers, 2) support marketing, sales or service processes, and 3) use wireless networks as the medium of delivery to the customer. Moreover, notice that in this paper we focus on CRM services which are offered to consumers (as opposed to businesses).

\section{An environment assessment framework}

In order to conduct our analysis of the mobile CRM landscape, we first refer to a framework, borrowed from [7], which suggests four relevant aspects to be considered in an assessment of a technology-based environment: the market, the value propositions, the actors and the issues. This allows us to get an overview of the mobile CRM context and direct our empirical investigation towards the most interesting aspects.

The basic idea is that the complex reality of an environment can be better understood by appraising it from various complementary perspectives focusing on distinct key aspects of the environment. The choice of perspectives was inspired by the remark of Porter that emergent industries experience major uncertainties at the levels of demand, actors' strategy and technology [24] and by the Balanced Scorecard with its consumer, business processes and innovation perspectives [22].

Four perspectives appear as essential in the analysis of a technology-based environment such as the mobile CRM landscape: the market, value propositions, actors and issues perspectives (see Figure 1).

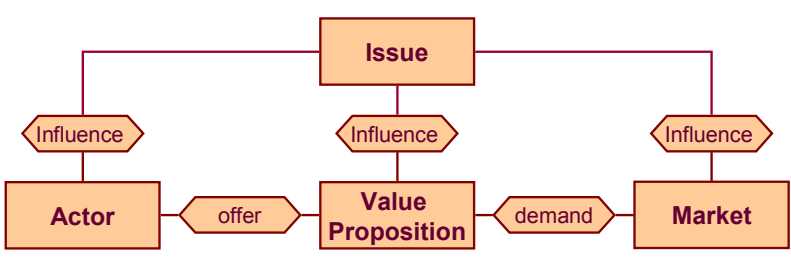

Figure 1: Environment assessment framework 
The market perspective deals with customers and demand uncertainties. Its analysis is vital because the success of any firm depends on its ability to create and maintain profitable relationships with customers by offering suitable value propositions. This entails investigating the demand side of the market in terms of who are the potential customers, what are their needs, how they generate desires to buy specific value propositions and result in an aggregate demand that can be addressed profitably [18]. Since customers tend to have distinct needs and preferences, it may be useful to group them into separate market segments [28].

The value proposition perspective focuses on the supply side of the market and deals with product innovation and competition. Analysis of the various value propositions offered in the market addressing the same user needs is crucial because their suitability to satisfy the needs determines their adoption by end customers. Moreover, innovative value propositions and advances in the underlying technologies must be monitored carefully as they may provide insights about future disruptive developments of the environment.

The actors perspective handles pressure relations between the various actors and strategic uncertainties. Its analysis comprises the various actors who can affect the industry's economic structure and competitive conditions, ultimately determining its profit potential. As suggested by Porter [24], these are primarily the existing and potential actors competing for the same customers (competitors, new entrants and substitute producers) or operating in adjacent industries along the value system (suppliers and distributors). Nevertheless, one should also consider other types of players which may more generally have an effect on environmental conditions or the preceding actors such as regulators, technology suppliers and the financial community [13].

Finally, the issues perspective deals with the major uncertainties which will determine the future evolution of the system under study. Issues can be defined as open questions, events, trends or other developments whose realization can greatly influence the future conditions of the environment and, consequently, the ability of the actors within it to achieve their objectives [2]. They may be source of opportunities and threats to the actors and can arise in areas such as the sociocultural, technologic, economic, ecologic and political domains [1]. Issues provide a good means to reflect on possible disruption of current conditions and the development of a broad set of evolution scenarios.

While we show them as separate, these perspectives are actually interdependent and linked to each other by influence relationships whereby elements in one perspective can influence elements in another. It is fundamental to consider these relationships as well.
The market and value propositions perspectives are tied by the fact that the market may adopt certain types of value propositions. While these adoption decisions may influence the type of value propositions, the offering of innovative value propositions may shape customer wants and thus affect adoption decisions.

Actors are simply linked to the value propositions that they develop and offer.

Finally, issues are potentially engaged in influence relationships with elements of all the other mentioned perspectives. These are bidirectional because while the outcome of the issue may exert an influence on the other elements, these other elements may in turn influence the outcome of the issue. In particular, actors and issues are linked in that actors may bias the outcome of some issues toward their preferences through their strategic action [3] and, conversely, in that the realization of certain issues can affect the ability of the various actors to attain their objectives. The market and issues are related by the fact that the outcome of some issues can affect market conditions, customer needs and adoption decisions At the same time, evolving market conditions may affect the realization of some issues. Finally, issues may influence value propositions, typically by affecting the evolution of the underlying technologies, while being at the same time affected by these developments.

\section{A snapshot of the current situation of the mobile CRM environment in Italy}

This section applies the previous framework to the mobile CRM services environment in Italy so as to get an idea of its general context and illustrates the results of our empirical study of its most relevant aspects.

\subsection{Data Collection Methodology}

The empirical study presented in this paper aims at providing an overview of the actual supply of mobile CRM services to Italian consumers. Accordingly, we designed our research as a comprehensive census of all existing offerings of mobile CRM services.

The first step of our research was to establish a methodology to identify these services and to collect information about them. In this respect, we dressed a large list of potentially useful information sources. A range of secondary source was first used to identify mobile CRM services. These comprised various Italian and international search engines and online directories, the web sites of mobile operators, service providers and the offering firms, newsletters, traditional media such as magazines and newspapers as well as trade shows. This information was then complemented by 
the insight obtained from a few primary sources via semi-structured interviews with various contact persons from mobile operators, offering firms and other mobile business related enterprises.

For each of the identified services we collected a number of data on the offering firm, its activity sector, its services, what is the underlying technologies, the way in which the service is activated and delivered to the customer, what are the technology partners, with what mobile operators is the service accessible, etc.

Finally remark that, consistent with our definition of mobile CRM services, we only considered in our study services that satisfy the following characteristics: 1) are not offered as stand-alone but are aimed at nurturing customer relationships, 2) support either marketing, sales or service processes, 3) use wireless networks as the medium of delivery and 4) are currently offered to consumers in the Italian market.

\subsection{Market}

We formerly stated that this paper aims at providing an overview of the supply of mobile CRM services to consumers. For that reason, and due to the difficulty to obtain demand side indicators in immature markets, we based our research on a survey of the supply side rather than focus directly on the demand side with consumer surveys. However, we are conscious that completing our findings with demand side insight is definitely important and this will be reminded in the concluding remarks about future research issues.

Although we did not focus on demand directly, from the impression gathered from the offering firms, it appears that there actually is some demand for mobile CRM services. In effect, some of the firms that we have interviewed have suggested that the initial usage of their mobile CRM initiatives was satisfactory.

Nevertheless, due to the novelty of such services and because of the reluctance of many firms to provide us information about the usage of their mobile CRM services, it is still premature to confirm our intuition and estimating their actual demand and adoption by end users by examining the supply side only.

\subsection{Value propositions}

With respect to the value proposition offered to consumers, our research has revealed that the mobile CRM market is beginning to attain a substantial size and presents good prospects for the future, at least from the supply viewpoint. In fact, our survey shows that the actual supply of mobile CRM services in the Italian market comprises as much as 750 services offered by 353 different firms. This represents a significant fraction of the global market of mobile services to consumers in Italy, which contains about 7000 services [5].

It is useful to distinguish the 750 services according to the type of service provided as shown in Figure 2.

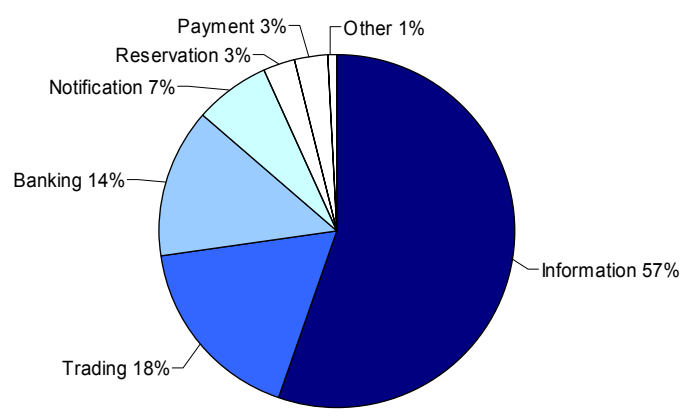

Figure 2: Repartition of services by type

The large majority of the 750 mobile CRM service is composed by the 422 information services, which account for the $57 \%$ of the total. These essentially include promotional and advertising messages as well as other useful information which is more or less related to the offering firm. Next are the 133 mobile trading services (18\%) and the 108 mobile banking services $(14 \%)$ services which allow the user to check its current status, perform operations and receive alerts. The remainder comprises 32 notification services (4\%), and 24 reservation services $(3 \%)$, mostly for tourism, tickets and goods, 20 payment services (3\%), mainly for parking lots, and 11 other services (1\%).

\subsection{Actors}

The mobile CRM value system comprises a number of key players which are directly or indirectly involved in the supply of mobile CRM services. These can be arranged into the five generic areas suggested by [8].

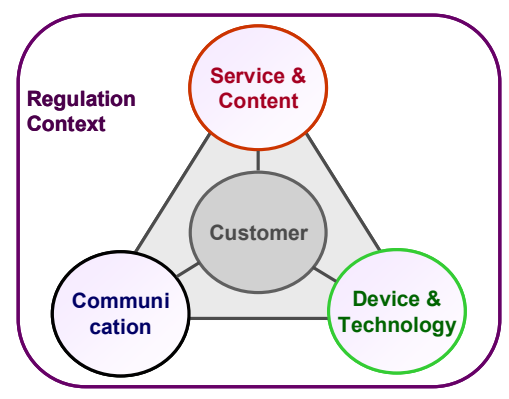

Figure 3: general mobile business key players

Customers are the obvious central point of the mobile CRM value system given that it is their 
adoption decisions that will ultimately determine the success of mobile CRM services.

In order to satisfy their needs for mobile CRM services, three families of actors are needed to provide the service itself, the communication infrastructure, and the device and other necessary technologies.

The service and content area constitutes the primary mobile CRM services value chain which is concerned with developing and providing the service itself. It comprises mobile CRM vendors, which provide the technology solutions underlying the service, wireless application service providers which manage and run the service in outsourcing, the firms which offer the service itself, and may eventually include content providers and aggregators as well.

As shown in Figure 4, mobile CRM services have not penetrated uniformly all industries. Our survey divided the 353 offering enterprises according to their industrial sector using the SIC classification. The largest group is formed by 131 public administrations including provinces and cities $(46 \%)$, followed by 76 service firms $(22 \%$, of which 42 in the entertainment and 21 in the tourism business), 61 financial businesses (17\%, mainly in the banking sector), 32 manufacturing firms (9\%), 19 transportation firms (5\%), 15 non-profit organizations $(4 \%), 5$ trade businesses $(1 \%)$, all 4 mobile operators $(1 \%)$ and 4 other organizations $(1 \%)$.

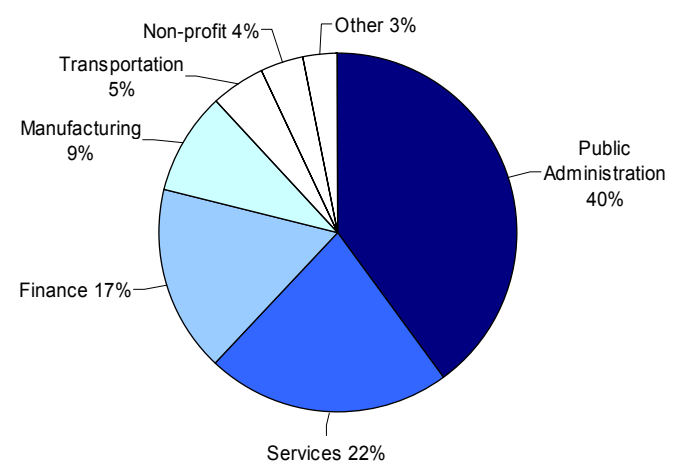

Figure 4: Repartition of actors by sector

Remark that the situation is similar at the European level, where despite the market is obviously at a early stage and the actual adoption is still fairly low, many interesting solutions have been recently developed tough not uniformly across all industries [4]. However, one particularity of the Italian market is the remarkably high penetration of public administrations.

In the communication area, the key actor are mobile network operators given that mobile CRM services are inevitably delivered through their wireless networks and messaging platforms. This puts them in a position to significantly preside over the whole value system and affect its expansion, mainly by setting the cost of transmissions. Operators also appear to play a role in branding the service, considered that only $44 \%$ of the services are independent from the carrier and $10 \%$ are offered in exclusivity by one of the four Italian mobile operators. In addition, wireless application service providers (WASP) may also play a role in providing a common interface between the firm and the various network operators.

The device and technology area essentially contains device and network equipment manufacturers. These are not actively involved in providing the service, but are very important as their choices will determine the features available to mobile CRM services and hence influence their future development and adoption.

Finally, the regulation and context area includes players such as regulation authorities, standardization bodies, financial community and user associations which may generally affect the mobile CRM context.

\subsection{Issues}

Before the potential benefits of mobile CRM can realize, a set of issues must be resolved. A lot of them are predictably related to technology and its actual limitations. However, as discussed by [29] issues related to applications, customers, business models and regulation must be taken into account as well.

With regard to technology, mobile CRM services may be delivered in various formats thanks to various technologies which can be based on messaging (SMS, MMS), browsing (WAP, i-mode), video streaming and applications download (J2ME, BREW). As shown in Figure 5, our survey indicates that the large majority of services are delivered in the form of SMS messages $(86 \%)$. A modest number of services $(23 \%)$ are available through browsing. Finally, almost inexistent are the services available with multimedia content using MMS (1\%) and video streaming (1\%).

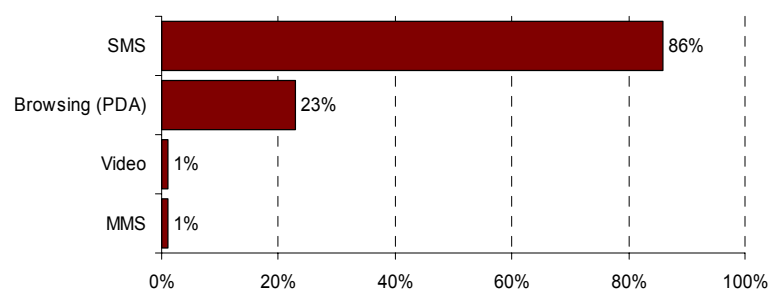

Figure 5: Repartition of services by delivery technology

The current situation in terms of technology is thus rather deceiving given that the predominant technology is SMS, which is the most limited medium of delivery as it can only convey a low amount of text. However, it is often sufficient for sending small but relevant pieces 
of information. The major reason is the low adoption of the newer generations of devices and networks, which limits the user base available to more advanced services such as those based on MMS or browsing with multimedia content. Fortunately, there is a marked trend towards the adoption of newer devices, which should encourage the future development of more compelling and presumably innovative services. As well, the proliferation of wireless LAN hotspots may also become a useful alternative delivery technology.

We also noticed a difference in the types of services provided through the various technologies: while in the case of SMS information services are the majority (56\%) followed by mobile banking and trading (31\%), in the case of browsing the situation is the opposite with mobile banking and trading accounting for $60 \%$ and information services for $36 \%$.

With reference to applications, content relevance, personalization and usability play a key role in mobile CRM since its goal is to develop individual customer relationships. Mobile devices are indeed very personal and customers expect an individualized experience and might get severely annoyed by even a few unwanted and useless messages. While all the concerned firms essentially agreed on this point, they are still struggling to find appropriate technical solutions.

Regarding customers and regulation, the respect of users' privacy is considered an issue which is critical for the adoption of mobile services. This essentially encompasses gaining the right to access and use user's personal data and avoiding mobile spam [6]. Mobile CRM services can be delivered upon the initiative of the firm without the customer specifically asking for it (push mode) or triggered by a specific customer (pull mode). While the existence of a previous relationship allows the firms to send CRM messages in a push mode, provided that they have the previous consent of the user (opt in) or they allow them to easily opt out, prudence in using push services is recommended given that there is a thin line between providing useful services and bombarding users with spam [6]. Indeed, one of the most challenging aspects is to reconcile users' demand for personalized services with their desire for privacy [25]. In our empirical study we found that $50 \%$ of the services are offered in a push mode (after the user has expressly activated the service, except for only $6 \%$ of them), $45 \%$ in a pull mode and $5 \%$ are available in both modes.

Finally one economic issue is the price of messages and communications charged by operators which may represent a major hindrance to mobile CRM. This cost may be sizeable and diminish the attractiveness of such services to the firm. As well, customers may not agree to pay to be offered or respond to such services.

\section{Conclusions}

In this paper, we presented a first snapshot of the current development of mobile CRM services to consumer obtained from an exhaustive survey of the current supply of mobile CRM services in the Italian market.

The main result obtained from our survey is that the mobile CRM services market is beginning to attain a substantial size, with about 750 services offered by 353 firms, and presents good future prospects at least from a supply viewpoint. This is a significant figure as compared with the about 7000 mobile services of all types offered to consumers in Italy.

The majority of the observed mobile CRM services is composed by the 422 information services which allow the transmission of promotional messages or other useful information related to the offering firm. The 133 mobile trading and 108 banking services also represent a significant portion, accounting for about a third of mobile CRM services. The remainder includes a small number of services supporting the reservation, notification and payment processes.

It is worth noticing that mobile CRM services have not uniformly penetrated all industries, but are mostly concentrated in public administrations, services and financial sectors, whilst surprisingly practically absent in the trade and manufacturing sectors.

However, a negative point is that the predominant delivery technology is SMS, mainly due to the low adoption of newer generations of devices with multimedia features. This is often sufficient for sending small but relevant pieces of information, but severely limits the types of services that can be offered. Fortunately, there is a marked trend towards the adoption of such newer devices, which should further encourage the future development of more compelling and presumably more innovative services.

We also briefly raised some other important issues such as personalization and privacy and we found that most providers are fortunately sensitive to these issues.

We believe that our survey gives a comprehensive and realistic picture of the current development of the supply of mobile CRM services in Italy. We also provided some insight about its potential evolution by examining some issues and their possible development.

We feel however that this is only a first step in the elaboration of a good picture of the actual situation of mobile CRM services and to future scenarios which could provide useful direction to the development of this promising sector. In particular, future research is required to deal with the highly important aspects of the demand side which were not treated in this paper. In that respect, instruments such as consumer surveys, 
focus groups or other market research techniques shall be employed to investigate end users needs and wants, their attitudes and intentions towards such services as well as their actual adoption decisions and usage. Furthermore, one limitation of this research is that our study is confined to the Italian market and therefore inherits all its peculiarities. Further studies in other settings may therefore provide other images of the mobile CRM landscape.

\section{Acknowledgements}

This research has been performed within the Mobile Value Added Services Observatory promoted by the School of Management of the Politecnico di Milano, Italy. The authors would like to provide credit to Gelpi Andrea and Groppo Diletta for the data collection efforts.

Moreover, the work presented in this paper was supported by the National Competence Center in Research on Mobile Information and Communication Systems (NCCR-MICS), a center supported by the Swiss National Science Foundation under grant number 5005-67322.

\section{References}

[1] K. R. Andrews, The concept of corporate strategy, 3rd ed. Dow Jones-Irwin, Homewood, 1987.

[2] H. I. Ansoff, "Strategic Issue Management", Strategic Management Journal, vol. 1, no. 2, 1980, pp. 131-148.

[3] J. Arcade, M. Godet, F. Meunier, and F. Roubelat, "Structural analysis with the MICMAC method \& Actor's strategy with MACTOR method." CNAM, 1999.

[4] M. Arnbjerg, "Emergence of Mobile CRM in Western Europe - Vendor Strategies", IDC 2004.

[5] U. Bertelè, A. Rangone, and F. Renga, "Mobile VAS: è boom multimediale", Dipartimento di Ingegneria Gestionale, Politecnico di Milano, Milano, Research Report, January 2005, 2005.

[6] G. Camponovo and D. Cerutti, "The Spam Issue in Mobile Business: a Comparative Regulatory Overview", presented at the Third International Conference on Mobile Business, New York, 2004.

[7] G. Camponovo, J. Ondrus, and Y. Pigneur, "Environmental Context Significance in Strategic Decision Support", presented at the International Workshop on Context Modeling and Decision Support, Paris, France, 2005.

[8] G. Camponovo and Y. Pigneur, "Analyzing the MBusiness Landscape", Annales des telecommunications, vol. 58, no. 1-2, 2003, pp. 59-77.

[9] J. Davies, D. Sepulcri, and E. Thompson, "CRM in Italy: A Market Appraisal and Overview", Gartner, 2003.

[10] A. Facchetti, A. Rangone, F. Renga, and A. Savoldelli, "Mobile marketing: an analysis of key success factors and the European value chain", International Journal of
Management and Decision Making, vol. 6, no. 1, 2005, pp. 65-80.

[11] D. Folinas, M. Vlachopoulou, V. Manthou, and D. Zogopoulous, "The value system of m-business", presented at the 1st International Conference on Mobile Business., Athens, 2002.

[12] H. Gebert, M. Geib, L. Kolbe, and W. Brenner, "Knowledge-Enabled Customer Relationship Management. Integrating Customer Relationship management and Knowledge Management Concepts", Journal of Knowledge Management, vol. 7, no. 5, 2003, pp. 107-123.

[13] M. Godet, Manuel de prospective stratégique, vol. 2, 2nd ed. Dunod, Paris, 2001.

[14] International Telecommunications Union, "Ubiquitous Network Societies: the Case of the Italian Republic", International Telecommunications Union forthcoming.

[15] S. Jarvenpaa, "Internet Goes Mobile: How Will Wireless Computing Affect Your Firm's Internet Strategy?", Center for Business, Technology and Law. University of Texas, 2000, pp. 21.

[16] R. Kalakota and M. Robinson, E-business 2.0 : roadmap for success. Addison-Wesley, Boston, MA, 2001.

[17] R. Kalakota and M. Robinson, M-Business: the race to mobility. McGraw-Hill, 2002.

[18] P. Kotler, Marketing management, 11th ed., Prentice Hall, Upper Saddle River, N.J, 2003.

[19] T. Levitt, "After the sale is over", Harvard Business Review, vol. 63, no. 5, 1983, pp. 87-93.

[20] Mobile Marketing Association, "What is Mobile Marketing", vol. 2004, 2004.

[21] F. Müller-Veerse, "Mobile Commerce Report", Durlacher 2000.

[22] D. P. Norton and R. S. Kaplan, The Balanced Scorecard: translating strategy into action, Harvard Business School Press, Boston, 1996.

[23] D. Peppers and M. Rogers, The one to one future: building relationships one customer at a time, 1 st ed. Currency Doubleday, New York, 1993.

[24] M. E. Porter, Competitive strategy: techniques for analyzing industries and competitors, The Free Press, New York, 1980.

[25] N. Sadeh, M Commerce: Technologies, Services, and Business Models, John Wiley \& Sons, 2002.

[26] D. Shani and S. Chalasani, "Exploiting Niches using Relationship Marketing", Journal of Consumer Marketing, vol. 9, no. 3, 1992, pp. 33-42.

[27] K. Siau, E.-P. Lim, and Z. Shen, "Mobile Commerce: Current States and Future Trends", in Advances in Mobile Commerce Technologies, E.-P. Lim and K. Siau, Eds. Hershey PA: Idea Group Pub., 2003, pp. 1-18.

[28] W. Smith, "Product Differentiation and Market Segmentation as Alternative Marketing Strategies", Journal of Marketing, vol. 21, 1956, pp. 3-8.

[29] P. Tarasewich, R. Nickerson, and M. Warkentin, "Issues in Mobile eCommerce", Communications of the AIS, vol. 8, 2002, pp. 41-64.

[30] Y. E. Zeng, H. J. Wen, and D. C. Yen, "Customer relationship management (CRM) in business-to-business (B2B) e-commerce", Information Management \& Computer Security, vol. 11, no. 1, 2003, pp. 39-45. 